\title{
A GENERAL COEFFICIENT THEOREM
}

BY

\author{
JAMES A. JENKINS
}

1. Teichmüller enunciated the principle that the solution of a certain type of extremal problem in geometric function theory is in general associated with a quadratic differential. If in the problem a point is assumed to be fixed without further requirement the quadratic differential will have a simple pole there. If in addition the functions of the class considered are required to have at the point, in terms of suitably assigned local uniformizing parameters, fixed values for their first $n$ derivatives, the quadratic differential will have a pole of order $n+1$ there. More generally, the highest derivative occurring may not be required to be fixed but some condition on its region of variation may be desired. Similar conditions apply to boundary points of a domain in which the class of functions considered is defined. Teichmüller was led to this principle by abstraction from the numerous results of Grötzsch [1-8] and by his considerations on quasiconformal mappings [26]. He applied this principle in certain concrete cases, the most important of which was his coefficient theorem [25] which is the most penetrating explicit result known in the general coefficient problem for univalent functions.

The object of the present paper is to prove a theorem which constitutes a precise formulation of the above principle for a wide class of extremal problems. At the end of the paper we shall discuss how this theorem includes the great majority of known results in geometric function theory.

2. We begin by collecting some definitions and known results.

We confine our attention to finite oriented Riemann surfaces. These are Riemann surfaces of finite genus which may have a finite number of hyperbolic boundary components. Such a surface is conformally equivalent to a domain lying on a closed oriented Riemann surface and bounded by a finite number of analytic curves.

On a finite oriented Riemann surface $\Re$ we recall the concept of quadratic differential. A quadratic differential is an entity which assigns to every local uniformizing parameter $z$ of $\Re$ a function $Q(z)$ meromorphic in the neighborhood associated with $z$ and satisfying the following conditions. If $z^{*}$ is a second local uniformizing parameter of $\Re$ whose neighborhood on $\Re$ overlaps that of $z$ and $Q^{*}\left(z^{*}\right)$ is the corresponding function associated with $z^{*}$, then at common points of the neighborhoods of $z$ and $z^{*}$ we have

$$
Q^{*}\left(z^{*}\right)=Q(z)\left(\frac{d z}{d z^{*}}\right)^{2} .
$$

Received by the editors June 8, 1953. 
In addition if $z$ is a boundary uniformizing parameter of $\Re$, the associated function $Q(z)$ is to be meromorphic on the closure of the semicircle in the $z$ plane corresponding to the neighborhood of $z$ on $\Re$, i.e. also on the boundary segment along the real axis. On the latter $Q(z)$ is to be real when defined, i.e. apart from poles. Naturally if $\Re$ is a closed surface this last condition becomes vacuous. From this definition it is clear that we may speak of a quadratic differential having a zero or pole of a given order at a point of $\Re$ or its boundary independently of the particular choice of local uniformizing parameter. A quadratic differential is to be denoted by the generic symbol $Q(z) d z^{2}$ or sometimes $d \zeta^{2}$.

Of great importance are the curves on which $Q(z) d z^{2}>0$. It is clear that these are independent of the choice of local uniformizing parameters. Maximal elements with this property will be called trajectories. They are either open arcs or topological circles on $\Re$. The totality of trajectories makes up a family $F$ which covers $\Re$ apart from the zeros and poles of the quadratic differential. By this we mean that through every other point of $\Re$ there passes a unique element of $F$. It is advantageous to group the zeros and simple poles in one set $C$ and the poles of order $\geqq 2$ in a second set $H$. Points in $C \cup H$ will be called singular points of $Q(z) d z^{2}$. The local structure of the trajectories in the neighborhood of any point of $\Re$ including those of $C$ and $H$ is discussed fully in [23, Chap. III] and summarized in $[17$, p. 8]. On this account we shall use the appropriate results without further explanation. We observe then that on $\Re$ punctured at the points of $H$ the family $F$ is much like the families considered in the topological theory of functions [16]. The principal difference is that the structure of $F$ in the neighborhood of points of $C$ is more general than that usually allowed in the theory mentioned. Nevertheless, natural generalizations of the methods used therein serve to establish various important results in the theory of quadratic differentials. Here, however, we shall use only some of the notions and terminology from [16].

Chief among these will be the notion of $F$-set. An $F$-set $K$ is defined here to be a subset of $\Re$ such that any trajectory which meets $K$ lies entirely in $K$. Also we will use the notion of inner closure. The inner closure of a set $K$ is defined to be the interior of the closure of $K$ and is denoted by $\widehat{K}$. It is readily verified that the inner closure of an $F$-set is an $F$-set [16].

In the sequel we shall be primarily concerned with the important special case in which the quadratic differential is regular on the boundary of $\Re$ and satisfies $Q(z) d z^{2} \geqq 0$. In this case we say we have a positive quadratic differential on $\Re$. Although we shall proceed without specifying whether $\Re$ has boundaries or not, we shall use the above term without qualification, understanding that if $\Re$ is closed the boundary requirement is vacuous.

Finally we shall make use also of the curves on which $Q(z) d z^{2}<0$. Maximal elements with this property will be called orthogonal trajectories.

3 . We wish now to discuss the structure of the family $F$ in the large, con- 
fining ourselves to the case of a positive quadratic differential. For this purpose we define some basic sets occurring in the decomposition to be given of $\Re[17]$.

An end domain $₹$ is a maximal connected open $F$-set on $\Re$ which is free from singular points, is swept out by trajectories each of which has a unique positive and negative limiting point at a given element $A$ of $H$, and is such that every neighborhood of $A$ contains trajectories in $\mathbb{F}$. An end domain has the property that the integral $\zeta=\int(Q(z))^{1 / 2} d z$ defined by analytic continuation from one local uniformizing parameter to another is single-valued in 5 and maps $\&$ on an upper or lower half-plane in the $\zeta$-plane. It turns out that $A$ must be a pole of order at least three.

A strip domain $\mathfrak{S}$ is a maximal connected open $F$-set on $\Re$ which is free from singular points, is swept out by trajectories each of which has one unique limiting end point at a first element $A$ of $H$ and another unique limiting end point at a second (possibly coincident) element $B$ of $H$, but is not an end domain. A strip domain has the property that the integral $\zeta$ $=\int(Q(z))^{1 / 2} d z$ is single-valued in $\subseteq$ and maps $\subseteq$ on a horizontal strip in the $\zeta$-plane.

A circle domain $\mathbb{S}$ is a maximal connected open $F$-set on $\Re$ containing exactly one element $A$ of $H$ and otherwise swept out by trajectories each of which is a topological circle. The element $A$ must be a pole of order 2 . If $c$ is a suitably chosen real constant the function

$$
\exp \left\{c \int(Q(z))^{1 / 2} d z\right\}
$$

maps $\mathbb{S}$ onto the interior of a circle.

A ring domain $\mathfrak{D}$ is a maximal doubly-connected open $F$-set on $\Re$, free from singular points, swept out by trajectories each of which is a topological circle and not contained in a circle domain. For suitable choice of $c$ it is mapped by the function (1) onto a circular annulus.

It is necessary to point out that for complete precision the above statements must be slightly modified. Consider, for example, the quadratic differential $d z^{2}$ on the $z$-sphere. It has only one singular point, a four-fold pole at infinity, and we can take the integral $\zeta$ to be identically $z$. The finite $z$-plane itself would come under the definition of end domain and thus not enjoy the mapping property stated. It is convenient to divide the $z$-plane along an arbitrary but fixed horizontal line and say that the two half-planes thus obtained are both end domains. There are a finite number of such exceptional cases which are easily enumerated, involving also the other types of basic domains. In each case suitable modifications are readily made and in order to avoid excessively complicated phraseology we do not give them explicit mention in the statement of the following Basic Structure Theorem and in the proof of the Main Theorem. We do, however, indicate their role in the 
question of equality in the Main Theorem.

We are now ready to state our

Basic Structure Theorem. Let $\Phi$ denote the union of all trajectories which have a limiting end point at a point of $C$. Then $\Re-\Phi$ consists of a finite number of end, strip, circle, and ring domains. Each such domain is bounded by a finite number of trajectories together with the points at which the latter meet. Every pole of order $m>2$ has a neighborhood covered by the inner closure of m-2 end domains and a finite number (possibly zero) of strip domains. Every pole of order 2 has a neighborhood covered by the inner closure of $a$ finite number of strip domains or has a neighborhood contained in a circle domain. $\Phi^{\widehat{T}}$ is an F-set consisting of a finite number of domains each of finite connectivity. Each boundary of such a domain is a piecewise analytic curve composed of trajectories and their limiting end points in $C$.

This theorem is essentially proved in $\$ 4$ of [17]. Although only hyperelliptic quadratic differentials are spoken of there, no use is made of this in the proof which applies to an arbitrary positive quadratic differential on a finite oriented Riemann surface. The first two statements are proved there explicitly. It may be mentioned that the exceptional cases indicated above arise when the image of $\Re$ by the integral $\zeta=\int(Q(z))^{1 / 2} d z$ is the simply covered $\zeta$-plane and $Q(z) d z^{2}$ has no simple pole. This point is not well clarified in [17]. The statements on the neighborhoods of points of $H$ follow at once from the nature of the basic domains and the fact that $\Phi$ is bounded away from points of $H[17$, p. 9]. The two final statements then follow as indicated in $\$ 7$ of [17]. Let it be remarked that while these statements were not made explicitly at that place they were used implicitly (and quite consciously) in the proof given there.

4. Let now a finite oriented Riemann surface $\Re$ be given with a fixed positive quadratic differential $Q(z) d z^{2}$ on it. By an admissible subdomain $\Delta$ of $\Re$ we mean a domain obtained by slitting $\Re$ along a finite number of arcs on trajectories of $Q(z) d z^{2}$ or on such trajectories closed by a point of $C$. Thus all points of $H$ are interior to $\Delta$ but points of $C$ may be on the boundary of $\Delta$.

We shall further consider a special class of mappings $f$ of $\Delta$ into $\Re$. First of all these mappings shall be homeomorphisms. Secondly as functions from one Riemann surface to another they shall be regular. Thus in a generalized sense they are univalent functions. Every pole contained in $\Delta$ is to be mapped into itself by $f$. Moreover at each point $A$ of $H$ we assign a fixed local parameter in terms of which $f$ is to have a prescribed local behavior. It is advantageous to choose a parameter $z$ such that $A$ is represented by the point at infinity in terms of $z$. If $A$ is a double pole of $Q(z) d z^{2}$, we require that in terms of this parameter we have locally

$$
f(z)=a z+a_{0}+a_{1} / z+\cdots, \quad a \neq 0 .
$$


If $A$ is a pole of order $m>2$, we require that in terms of this parameter we have locally

$$
f(z)=z+\frac{a_{m-3}}{z^{m-3}}+\cdots .
$$

The same conditions are readily expressed in terms of an ordinary local uniformizing parameter.

Instead of fixing a single local parameter at $A$ it would be enough to fix a class of parameters related to one another in the manner

$$
\tilde{z}=z+\frac{b_{m-2}}{z^{m-2}}+\cdots
$$

at a pole of order $m \geqq 2$, the parameters being conditioned as above. In this way we would obtain from $\Re$ a "higher principal domain" in the sense of Teichmüller [27].

Finally we require that $f$ be homotopic to the identity in the following sense. There shall exist a function $F(P, t)$ defined for $P \in \Delta, 0 \leqq t \leqq 1$, with values in $\Re$, continuous in both variables together, satisfying

$$
\begin{aligned}
& F(P, 0)=f(P), \\
& F(P, 1)=P, \\
& F(P, t)=P, P \text { a pole in } \Delta, \\
& F(P, t) \neq Q, Q \text { a pole in } \Re, P \neq Q, t \text { arbitrary. }
\end{aligned}
$$

A homotopy of this type will be called an admissible homotopy. A function $f$ satisfying the above conditions will be called an admissible function on $\Delta$.

We are now ready to state our

MAIN ThEOREM. Let $\Re$ be a finite oriented Riemann surface, $Q(z) d z^{2} a$ positive quadratic differential on $\Re, \Delta$ an admissible subdomain of $\Re$ (relative to $\left.Q(z) d z^{2}\right)$, and $f$ an admissible function on $\Delta$. Let $Q(z) d z^{2}$ have double poles $P_{1}, \cdots, P_{r}$ and poles $P_{r+1}, \cdots, P_{n}$ of order $>2$. We allow either of these sets to be void but not both. At $P_{j}, j \leqq r$, in terms of a parameter $z$ conditioned as above let $f$ have the expansion

$$
f(z)=a_{z}^{(i)}+a_{0}^{(j)}+\frac{a_{1}^{(i)}}{z}+\cdots
$$

and $Q$ the expansion

$$
Q(z)=\frac{\alpha^{(j)}}{z^{2}}+\text { higher powers of } z^{-1} .
$$

At $P_{j}, j>r, a$ pole of order $m_{j}>2$, let us have the corresponding expansions 


$$
\begin{aligned}
& f(z)=z+\frac{a_{m_{j}-3}^{(j)}}{z^{m_{j}-3}}+\cdots \\
& Q(z)=\alpha^{(j)} z^{m_{j}-4}+\text { decreasing powers of } z .
\end{aligned}
$$

Then

$$
\Re\left(\sum_{j=1}^{r} \alpha^{(j)} \log a^{(j)}+\sum_{j=r+1}^{n} \alpha^{(j)} a_{m_{j-3}}^{(j)}\right) \leqq 0 .
$$

Equality can be attained in (2) only for $f$ the identity in the following circumstances.

(i) There is a pole of order $>2$.

(ii) There is a pole $P_{j}, j \leqq r$, of order 2 with corresponding coefficient $a^{(j)}=1$.

(iii) There is a simple pole or a point on a trajectory ending in a simple pole in $\Delta$.

Equality can occur with $\left|a^{(j)}\right| \neq 1, j \leqq r$, for $\alpha^{(j)}<0$ only when $\Re$ is the sphere and $Q(z) d z^{2}$ a quadratic differential whose only singular points are two poles of order 2. Then $f$ may be an arbitrary linear transformation with these as fixed points.

Examples of equality occurring for nonidentical $f$ in other cases can be constructed with almost arbitrary degrees of complexity.

To make the statement of the theorem complete it is necessary to give the determination of the logarithms in our fundamental inequality (2). Under the deformation $F$ a given point $P \in \Delta$, not a pole, describes a path $F(P, t)$, $0 \leqq t \leqq 1$. If $P$ is sufficiently close to a second order pole $P_{j}$ this path will lie in the neighborhood of the parameter $z$ associated with $P_{j}$. The change in argument of the value of $z$ corresponding to $F(P, t)$ as $t$ runs from 0 to 1 tends to a limit $-\theta_{j}$ as $P$ approaches $P_{j}$, where $\theta_{j}$ is one value of the argument of $a^{(j)}$. Then we understand

$$
\log a^{(j)}=\log \left|a^{(j)}\right|+i \theta_{j}
$$

to hold in (2).

Basic in our proof is the use of the metric $|d \zeta|=|Q(z)|^{1 / 2}|d z|$ which is a conformally invariant metric on the surface $\Re$. It will frequently be referred to as the $Q$-metric. In the metric space thus obtained from $\Re$ the points of $H$ are to be excluded, being at "infinite distance."

5. We shall now collect a number of fundamental results due to Teichmüller which will be used in our proof.

First we recall the simplifying remark that we may assume that the quadratic differential has no simple poles on $\Re[26$, p. 160] (see also [23, Chap. VII]). Indeed since there is at least one element in $H$ we can replace $\Re$ by a two-fold covering surface $\Re^{*}$, doubly branched at the simple poles of the quadratic differential and possibly at one pole of higher order. The positive 
quadratic differential, an admissible subdomain, and an admissible function induce similar entities in a natural fashion on $\Re^{*}$. In terms of these the lefthand side of (2) is replaced by just twice that expression. Thus it is enough to prove the fundamental inequality (2) on $\Re^{*}$. To discuss equality we return to the surface $\Re$ in a manner which will be clear later. Until then we shall assume that the present situation holds for $\Re$ itself. Actually the argument to follow can be carried out with proper conventions even when simple poles are present but it complicates some of the arguments.

Fundamental for the following is the consideration of the geodesics in the $Q$-metric on $\Re$. By a geodesic we understand as usual a curve of which every sufficiently small subarc is the shortest join of its end points. Locally an arc is a geodesic if on it is satisfied

$$
\arg Q(z) d z^{2}=\text { constant }
$$

except at a zero of the quadratic differential. Two arcs satisfying (3) and meeting at an $m$-fold zero of $Q(z) d z^{2}$ make up a geodesic arc if and only if each of the angles they form is at least $2 \pi /(m+2)$.

Let now $A$ and $B$ be any two points of $\Re-H$. We regard the homotopy classes of curves in $\Re-H$ joining $A$ and $B$ in the sense that the end points are to be fixed at $A$ and $B$ during the deformation. We then have the result due to Teichmüller $[26, \S 138]$.

I. In every homotopy class of curves in $\Re-H$ joining $A$ and $B$ there is a unique geodesic which is the shortest join of $A$ and $B$ among curves of the homotopy class.

Also we have

II. Let $\Delta$ be an admissible subdomain of $\Re, K$ a compact subset of $\bar{\Re}-H$. Let $\Lambda$ be a subarc of a trajectory in $\Delta \cap K$ and $\Lambda^{\prime}$ its image under $f$, an admissible mapping on $\Delta$. Then there exists a real constant $M$ depending on $K$ but not on $\Lambda$ such that

$$
\int_{\mathbf{\Lambda}^{\prime}}|d \zeta| \geqq \int_{\Lambda}|d \zeta|-M .
$$

The present situation is slightly different from that considered by Teichmüller but his proof $[26, \S 136]$ goes over almost word for word so is not repeated here. By $\bar{\Re}$ we naturally mean $\Re$ together with its boundaries (if any). Finally

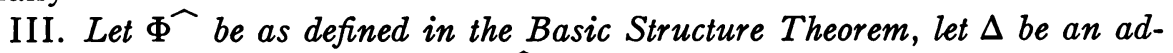

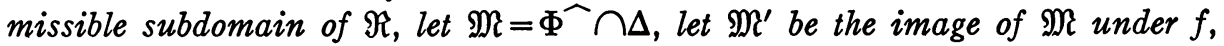
an admissible mapping on $\Delta$, and let $d A(P)$ denote the element of area at $P$ in the Q-metric. Then

$$
\iint_{\mathfrak{M},} d A \geqq \iint_{\mathfrak{M}} d A
$$


Once again the situation is slightly different from that considered by Teichmüller but this time we shall give the proof, even though it differs very little from that of Teichmüller [26, \$135], in order to bring out the essential properties of $\Phi \widehat{ }$ on which the result depends.

We consider then $\mathfrak{M}$ and the mapping $f$. By $\left|f^{\prime}(P)\right|$ we mean the distortion produced by $f$ at the point $P$ in terms of the $Q$-metric. Now on the trajectory through $P \in \mathfrak{M}-C$ we draw in each direction a segment of length $L / 2$ in the $Q$-metric lying in $\mathfrak{M}(L>0)$. This is possible for all values of $L$ unless $P$ lies on a trajectory ending at a point of $C$ or on one which has been slit along an arc to obtain $\Delta$ from $\Re$. Since there is only a finite number of each kind it proves we can neglect them. We denote arc length measured algebraically from $P$ on the arc so obtained by $t$ and write the curve in parametric form as $S(t, P)(-L / 2 \leqq t \leqq L / 2) . S(t, P)$ is a point of $\mathfrak{M}$ and $S(0, P)=P$.

From II we obtain at once

$$
\int_{-L / 2}^{L / 2}\left|f^{\prime}(S(t, P))\right| d t \geqq L-M .
$$

We integrate this over $\mathfrak{M}$ with respect to area in the $Q$-metric to get

$$
\iint_{\mathfrak{R}} \int_{-L / 2}^{L / 2}\left|f^{\prime}(S(t, P))\right| d t d A(P) \geqq(L-M) \iint_{\mathfrak{M}} d A .
$$

Now on the left-hand side we take $|t|, S$ as independent variables in place of $t, P$ noting that

$$
d t d A(P)=d|t| d A(S)
$$

and changing the order of integration we obtain

$$
2 \iint_{\mathfrak{M}}\left|f^{\prime}(S)\right| d A(S) \int_{0}^{L / 2} d|t| \geqq(L-M) \iint_{\mathfrak{M}} d A
$$

or

$$
L \iint_{\mathfrak{R}}\left|f^{\prime}(S)\right| d A(S) \geqq(L-M) \iint_{\mathfrak{M}} d A .
$$

Dividing by $L$ and letting it approach $\infty$ we obtain

$$
\iint_{\mathfrak{M}}\left|f^{\prime}(S)\right| d A(S) \geqq \iint_{\mathfrak{M}} d A .
$$

An obvious application of Schwarz's inequality gives

$$
\iint_{\mathfrak{R}}\left|f^{\prime}(S)\right|^{2} d A(S) \geqq \iint_{\mathfrak{M}} d A,
$$


thus

$$
\iint_{\mathfrak{M}^{\prime}} d A \geqq \iint_{\mathfrak{R}^{\prime}} d A
$$

as stated.

Further it follows that equality is possible only if the mapping is everywhere on $\mathfrak{M}$ isometric in the $Q$-metric.

We point out the two essential properties used in this proof : first closure $\Phi$ is compact on $\bar{\Re}-H$, second $\Phi$ is an $F$-set.

6. We are now ready to begin the proof proper of the Main Theorem. A general outline of this is as follows. First we must remove from $\Delta$ suitably fashioned neighborhoods of the points of $H$. Then we obtain evaluations of the area of the residual domain and its image under $f$, one from above using the behavior of $f$ on the boundaries of the neighborhoods removed, one from below using the method of Grötzsch. The combination of these gives the desired inequality (2).

First we describe the neighborhoods to be removed. Let $P_{j} \in H, j>r$, be a pole of $Q(z) d z^{2}$ of order $m_{j}>2$. Let $U$ be a neighborhood of $P_{j}$ covered by the inner closure of the $m_{j}-2$ end domains and possible strip domains associated with $P_{j}$. We slit this neighborhood along a trajectory $\Lambda$ running to the point $P_{j}$ and in the other sense leaving $U$, say a boundary curve of one of the end domains. In the set $U-\bar{\Lambda}, \zeta=\int(Q(z))^{1 / 2} d z$ is single-valued (choosing any particular determination) and it maps this set on a portion of Riemann surface over the $\zeta$-plane. We regard in the $\zeta$-plane a square of side $2 L$, center at the origin, and with sides parallel to the real and imaginary axes. We take the trace of this square on the Riemann surface and its inverse image on $\Re$. The latter is a sequence of arcs as follows. First there is an arc of an orthogonal trajectory with initial point on $\Lambda$. Then there is an arc of a trajectory lying entirely in an adjacent end domain (for $L$ large enough). Next there is an arc of an orthogonal trajectory. They alternate in this manner until we reach an arc of an orthogonal trajectory ending on $\Lambda$. Its terminal point will not in general coincide with the initial point of the first arc above. Joining these points with an arc of $\Lambda$ if necessary we obtain a simple closed curve which we denote by $\gamma\left(P_{j}, L\right)$. It divides $\Re$ into two components and the one which contains $P_{j}$ is the neighborhood to be removed. It will be denoted by $U\left(P_{j}, L\right)$.

Now let $P_{j} \in H, j \leqq r$, be a pole of $Q(z) d z^{2}$ of order 2 . There are two cases according as $P_{j}$ lies in a circle domain or not. In the first case we slit a neighborhood $U$ of $P_{j}$ lying in the circle domain along an orthogonal trajectory $N$ running from the boundary of $U$ to $P_{j}$. In the set $U-\bar{N}, \zeta=\int(Q(z))^{1 / 2} d z$ is single-valued (again any particular determination) and it maps this set on a vertical strip in the $\zeta$-plane. We may suppose that $\Im \zeta$ becomes positively in- 
finite in the strip. Then we take the intersection of the strip with the line $\Im \zeta=L$ (where $L$ will be a large positive number). Corresponding to this on $\Re$ will be a closed trajectory in the circle domain which we denote by $\gamma\left(P_{j}, L\right)$. This divides $\Re$ into two components and the one which contains $P_{j}$ is the neighborhood to be removed. It will be denoted by $U\left(P_{j}, L\right)$.

Finally let $P_{j}$ be a pole of order 2 which has a neighborhood $U$ lying in the inner closure of a union of strip domains. We slit $U$ along a trajectory $\Lambda$ running from its boundary to $P_{j}$. In the set $U-\bar{\Lambda}, \zeta=\int(Q(z))^{1 / 2} d z$ is singlevalued (any particular determination) and it maps this set on a horizontal strip in the $\zeta$-plane. We may suppose that $\Re \zeta$ becomes positively infinite in the strip. Then we take the intersection of the strip with the line $\Re \zeta=L$ ( $L$ again large and positive). Corresponding to this on $\Re$ will be an arc of an orthogonal trajectory with end points on $\Lambda$. These will not in general be coincident. Joining them with an arc of $\Lambda$ if necessary we obtain a simple closed curve which we denote by $\gamma\left(P_{j}, L\right)$. It divides $\Re$ into two components and the one which contains $P_{j}$ is the neighborhood to be removed. It will be denoted by $U\left(P_{j}, L\right)$.

Removing all the neighborhoods $U\left(P_{j}, L\right), j=1, \cdots, n$, from $\Delta$ we obtain a domain $\Delta(L)$ which is the domain we shall treat. It should be observed that $\Delta(L)$ depends not only on the parameter $L$ but also on the various choices of the determination of $\zeta=\int(Q(z))^{1 / 2} d z$ in the neighborhoods of the points of $H$. However we shall keep one fixed determination for every point of $H$ and thus $\Delta(L)$ will be a well defined domain for given $L$. In the sequel we shall always suppose $L$ so large that no slit boundary of $\Delta$ meets any $\gamma\left(P_{j}, L\right)$ or penetrates into the interior of any $U\left(P_{j}, L\right)$.

We now compare the area of $\Delta(L)$ in the $Q$-metric with that of its image $\Delta^{\prime}(L)$ under $f$. From the form of $\Delta(L)$ and the properties of $f$ it is clear that each of these is finite. Our first step is to estimate the area of $\Delta^{\prime}(L)$ from above by the area contained by the image curves of the curves $\gamma\left(P_{j}, L\right)$, i.e. the area of the domain on $\Re$ having these images as boundaries. For this it is enough to determine the change in area arising from each such boundary.

Let first $P_{j} \in H, j>r$, be a pole of order $m_{j}>2$. Under $\zeta=\int(Q(z))^{1 / 2} d z$ there corresponds to the curve in question a finite sequence of segments differing from $m_{j}-2$ half-boundaries of squares by at most two vertical and one horizontal segment, each of fixed length. To determine the resultant change in area we shall find the mapping induced in the $\zeta$-plane by the mapping $f$. First we observe

$$
\zeta=\alpha^{1 / 2}\left(\frac{m}{2}-1\right)^{-1} z^{m / 2-1}+\text { decreasing powers of } z^{1 / 2}
$$

where we have written $\alpha, m$ for $\alpha^{(j)}, m_{j}$ and for $m$ even there may be a logarithmic term in the expansion. The choice of roots appearing depends on the determination of $\zeta$. Let $\omega$ be the point corresponding to $\zeta$ under the mapping 
induced by $f$. Then

$$
\begin{aligned}
\omega & =\zeta+\left(\alpha^{1 / 2} z^{m / 2-2}+\cdots\right)\left(\frac{a_{m-3}}{z^{m-3}}+\cdots\right) \\
& =\zeta+\alpha a_{m-3}\left(\frac{m}{2}-1\right)^{-1} \zeta^{-1}+O\left(|\zeta|^{-(1+k)}\right)
\end{aligned}
$$

where $k=2 /(m-2)$ and we have written $a_{m-3}$ for $a_{m-3}^{(j)}$. Thus the change in area arising from the effect of the mapping on the half-boundary of a square is

$$
\Re\left\{\frac{1}{2 i} \int\left(\bar{\zeta}+b \xi^{-1}\right)\left(1-b \zeta^{-2}\right) d \zeta-\frac{1}{2 i} \int \xi d \zeta\right\}+O\left(L^{-k}\right)
$$

where the integrals are taken on the half-boundary of the square and we have written $b$ for $\alpha a_{m-3}(m / 2-1)^{-1}$. The whole expression is seen at once to be $O\left(L^{-k}\right)$. As for the change arising from the segments mentioned above it is clearly $O\left(L^{-1}\right)$. Thus the total change is $O\left(L^{-1}\right)$ or $O\left(L^{-k}\right)$ whichever is larger. In any case the change in area arising from the effect of $f$ on $\gamma\left(P_{j}, L\right), j>r$, is $o(1)$ which is all we need.

Now let $P_{j} \in H, j \leqq r$, be a pole of order 2 lying in a circle domain. It is clear that this corresponds to having $\alpha^{(j)}<0$. Under $\zeta=\int(Q(z))^{1 / 2} d z$ corresponds to $\gamma\left(P_{j}, L\right)$ a horizontal segment of length $2 \pi\left|\alpha^{(j)}\right|^{1 / 2}$. To determine the resultant change in area we find the mapping induced in the $\zeta$-plane by the mapping $f$. First we have

$$
\zeta=\alpha^{1 / 2} \log z+\text { powers of } z^{-1}
$$

where we have written $\alpha$ for $\alpha^{(j)}$. The choice of determination previously made for $\zeta$ implies that this root $\alpha^{1 / 2}$ has positive imaginary part. (It is pure imaginary.) Let $\omega$ denote the point corresponding to $\zeta$ under the mapping induced by $f$. Then

$$
\omega=\zeta+\alpha^{1 / 2} \log a^{(j)}+\text { terms in } z^{-1} .
$$

The image domain has side boundaries corresponding to $N$ which are obtained from one another by a translation of $2 \pi|\alpha|^{1 / 2}$. Thus the change in area is $2 \pi|\alpha|^{1 / 2} \Im\left(\alpha^{1 / 2} \log a^{(j)}\right)+o(1)=-2 \pi \Re\left(\alpha \log a^{(j)}\right)+o(1)$.

Finally let $P_{j} \in H, j \leqq r$, be a pole of order 2 not lying in a circle domain. Under $\zeta=\int(Q(z))^{1 / 2} d z$ to $\gamma\left(P_{j}, L\right)$ there correspond a vertical segment and a horizontal segment (the latter possibly degenerating to a point), each of constant length. They have one end point in common and the segment joining their other end points corresponding to a circuit about $P_{j}$ leaving $P_{j}$ to the right is given by $2 \pi i\left(\alpha^{(j)}\right)^{1 / 2},\left(\alpha^{(j)}\right)^{1 / 2}$ to have positive real part corresponding to the choice of determination of $\zeta$ previously made. Once again

$$
\zeta=\alpha^{1 / 2} \log z+\text { powers of } z^{-1}
$$


with the same simplified notation as before. Letting $\omega$ denote the point corresponding to $\zeta$ under the mapping induced by $f$ we have

$$
\omega=\zeta+\alpha^{1 / 2} \log a^{(j)}+\text { terms in } z^{-1} .
$$

In case $\alpha$ is not real and positive we now take the neighborhood of $P_{j}$ slit not along a trajectory but along an arc meeting the trajectories at equal angles and having a limiting direction at $P_{j} . \zeta$ will map this on a region with sides parallel to $\alpha^{1 / 2}$ and of constant width $2 \pi|\alpha|^{1 / 2}$. Then as before we get for the change in area $2 \pi \Re\left(|\alpha| \log a^{(j)}\right)+o(1)$. In the above considerations we may for definiteness assume $\log a^{(j)}$ to have the determination given following the statement of the Main Theorem but we see that the determination plays no role in the present final result.

If we divide the poles of order 2 according to the above criterion into $P_{1}, \cdots, P_{s}$, lying in circle domains, $P_{s+1}, \cdots, P_{r}$ not (where we understand that either or both of these sets may be void) we have our first evaluation:

$$
\begin{aligned}
\iint_{\Delta^{\prime}(L)} d A \leqq \iint_{\Delta(L)} d A & -\sum_{j=1}^{s} 2 \pi \Re\left(\alpha^{(j)} \log a^{(j)}\right) \\
& +\sum_{j=8+1}^{r} 2 \pi \Re\left(\left|\alpha^{(j)}\right| \log a^{(j)}\right)+o(1)
\end{aligned}
$$

where again $d A$ denotes the element of area in the $Q$-metric.

7. We next estimate the area of $\Delta^{\prime}(L)$ in the $Q$-metric from below, making use of Grötzsch's method. We now interpret this quantity as the area of $\Delta(L)$ in a new metric, namely $\left|f^{\prime}(P)\right||d \zeta(P)|$ where the derivative has the same meaning as in $\S 5$. We denote this for brevity by $\rho|d \zeta|$. It is clear that

$$
\iint_{\Delta(L)} \rho^{2} d A=\iint_{\Delta^{\prime}(L)} d A .
$$

Now $\Delta(L)$ is decomposed into $\mathfrak{M}=\Phi^{\wedge} \cap \Delta$, the union $\mathfrak{D}^{*}$ of a finite number of intersections of ring domains with $\Delta$ and the union of a finite number of intersections of end, strip, and circle domains with $\Delta(L)$.

The result III shows that

$$
\iint_{\mathfrak{R}} \rho^{2} d A \geqq \iint_{\mathfrak{M}} d A
$$

and quite similarly

$$
\iint_{\mathfrak{D}^{*}} \rho^{2} d A \geqq \iint_{\mathfrak{D}^{*}} d A .
$$

Further if $\mathfrak{S}(L)$ denotes the intersection of a circle domain $\sqrt{5}$ with $\Delta(L)$ we have again 


$$
\iint_{\mathfrak{C}(L)} \rho^{2} d A \geqq \iint_{\mathfrak{C}(L)} d A \text {. }
$$

Let now $₹$ be an end domain adjacent to a pole $P_{j}, j>r$, of order $m_{j}=m$ for simplicity, and let $\mathbb{E}(L)=\Subset \cap \Delta(L)$. First we take the extended domain $F^{*}$ of $\mathbb{F}$ in the sense of Schaeffer and Spencer [23, pp. 80, 97]. This is a simply-connected subset of $\Re$ containing $₹$ which is mapped by the given determination of $\zeta$ on the entire $\zeta$-plane slit along a finite number of vertical half-infinite segments each starting at the image of a zero and not entering the image of $\&$. We denote the $\zeta$-plane so slit by $E$.

Now

$$
0 \leqq \iint_{\mathbb{E}(L)}(\rho-1)^{2} d A=\iint_{\mathscr{E}(L)} \rho^{2} d A+\iint_{\mathscr{E}(L)} d A-2 \iint_{\mathfrak{E}(L)} \rho d A .
$$

Setting $\zeta=\xi+i \eta$ and passing to the $\zeta$-plane we have

$$
\iint_{E(L)} \rho d A=\iint_{E(L)} \rho d \xi d \eta \text {. }
$$

The range of integration on the right-hand side is the image $E(L)$ of $\mathbb{E}(L)$, a rectangle of horizontal dimension $2 L$ and of vertical dimension $L+\lambda$ where $\lambda$ is fixed, real, possibly either positive or negative. Let us suppose for definiteness that $\Im \zeta$ becomes positively infinite in $E(L)$ as $L$ approaches infinity. $E(L)$ then differs from the half square $-L \leqq \xi \leqq L, 0 \leqq \eta \leqq L$ at most by a horizontal strip of finite width.

Let $\sigma(\eta)$ denote the horizontal segment of length $2 L$ lying in $E(L)$ on the line at height $\eta$. This will exist for all but a finite number of $\eta$ in $L \geqq \eta \geqq-\lambda$ (i.e. unless we encounter the image of a boundary slit of $\Delta(L)$ ). Then $\int_{\sigma(\eta)} \rho d \xi$ is just the length in the $Q$-metric of the image $\tau$ under $f$ of the antecedent of $\sigma(\eta)$ by the mapping $\zeta$. Since $f$ is continuous at $P_{j}$ the images $A$ and $B$ of the end points of $\sigma(\eta)$ under the mapping in the $\zeta$-plane induced by $f$ will lie in $E$ when $L$ is large enough. A similar remark applies to the images by $\zeta$ of the paths described under the homotopy $F$ by the end points of the antecedent of $\left.\sigma(\eta){ }^{1}\right)$. Consider the shortest polygonal line lying in $E$ and joining $A$ and $B$. Clearly it is the image by $\zeta$ of a geodesic on $\bar{\Re}$. A standard device shows that this geodesic is in the same homotopy class as $\tau$. Thus $\int_{\sigma(\eta)} \rho d \xi$ is estimated from below by the difference of the values of $\xi$ at $A$ and $B$. Since the mapping induced by $f$ is given by

$$
\omega=\zeta+\alpha a_{m-3}\left(\frac{m}{2}-1\right)^{-1} \zeta^{-1}+O\left(|\zeta|^{-(1+k)}\right)
$$

(1) For the complete justification of this statement it may be necessary to modify the deformation $F$ in a neighborhood of $P_{j}$. This can be done but a better method is to avoid this question by the use of a suitable direct homotopy argument. 
we have, taking the current point on the right-hand vertical side of $E(L)$ to be $L+i L \tan \theta$ and writing $\alpha a_{m-3}(m / 2-1)^{-1}=M e^{i \mu}$,

$$
\int_{\sigma(\eta)} \rho d \xi \geqq 2 L+2 M L^{-1} \cos \mu \cos ^{2} \theta+O\left(L^{-(1+k)}\right) .
$$

Integrating this over the range of $\eta$ corresponding to $E(L)$ we get (there being only a finite number of exceptional values of $\eta$ )

$$
\iint_{\mathbb{E}(L)} \rho d A \geqq 2 L(L+\lambda)+2 M L^{-1} \cos \mu \int_{\theta_{0}}^{\pi / 4} \cos ^{2} \theta d L \tan \theta+O\left(L^{-k}\right)
$$

where $\theta_{0}$ is the value of the angle $\theta$ corresponding to the lower bound of integration. Replacing it by 0 changes that term only by an amount $O\left(L^{-1}\right)$. Thus

$$
\iint_{\mathfrak{E}(L)} \rho d A \geqq \iint_{\mathfrak{E}(L)} d A+\pi(m-2)^{-1} \Re\left(\alpha a_{m-3}\right)+o(1) .
$$

Since there are $m-2$ end domains associated with a pole of order $m$ and $\iint_{\mathfrak{E}(L) \rho^{2}} d A+\iint_{\mathfrak{E}_{(L)}} d A \geqq 2 \iint_{\mathfrak{E}_{(L)} \rho d A}$ we have

$$
\sum \iint_{\mathfrak{E}_{(L)}} \rho^{2} d A \geqq \sum \iint_{\mathfrak{E}_{(L)}} d A+2 \pi \Re\left\{\sum_{j=r+1}^{n} \alpha^{(j)} a_{m_{j-3}}^{(j)}\right\}+o(1),
$$

the first two summations being taken over the totality of end domains.

Next let $\subseteq$ be a strip domain and let $\subseteq(L)=\subseteq \cap \Delta(L)$. As before we have

$$
\iint_{\varsigma_{(L)}} \rho^{2} d A+\iint_{\varsigma_{(L)}} d A \geqq 2 \iint_{\varsigma_{(L)}} \rho d A
$$

and the right-hand side can be evaluated from below as before using in the argument the corresponding extended domain $[23$, p. 80$]$ and geodesics. It is verified at once that the increment of $\iint_{\varsigma_{(L)} \rho d A}$ over $\iint_{\varsigma_{(L)}} d A$ accruing from a pole of order $>2$ is $o(1)$ while the increment to the totality of $\iiint_{(L)} \rho d A$ for $\subseteq$ ending at a given pole $P_{j}, s+1 \leqq j \leqq r$, of order 2 is $2 \pi\left|\alpha^{(j)}\right|^{1 / 2} \cos \phi_{j}$ $\Re\left\{\left(\alpha^{(j)}\right)^{1 / 2} \log a^{(j)}\right\}+o(1)$ where $\left(\alpha^{(j)}\right)^{1 / 2}$ is the root with positive real part, $\left(\alpha^{(j)}\right)^{1 / 2}=\left|\alpha^{(j)}\right| 1 / 2 e^{i \phi_{i}}$, and $\log a^{(j)}$ has the determination previously given. (This is the only place where the choice of determination of $\log a^{(i)}$ has an actual effect.)

Combining these results for all strip domains we find

$$
\begin{aligned}
\sum \iint_{\Phi_{(L)}} \rho^{2} d A \geqq & \sum \iint_{\Phi_{(L)}} d A \\
& +4 \pi \Re\left\{\sum_{j=8+1}^{r}\left|\alpha^{(j)}\right| e^{i \phi_{j}} \cos \phi_{j} \log a^{(j)}\right\}+o(1)
\end{aligned}
$$

where the first two summations are taken over all strip domains. 
Adding up the results given for all the domains into which $\Delta(L)$ is decomposed we obtain

$$
\begin{aligned}
\iint_{\Delta(L)} \rho^{2} d A \geqq \iint_{\Delta(L)} d A & +4 \pi \Re\left\{\sum_{j=s+1}^{r}\left|\alpha^{(j)}\right| e^{i \phi_{j}} \cos \phi_{j} \log a^{(j)}\right\} \\
& +2 \pi \Re\left\{\sum_{j=r+1}^{n} \alpha^{(j)} a_{m_{j-3}}^{(j)}\right\}+o(1)
\end{aligned}
$$

which is our second evaluation.

8. Combining the two evaluations, recalling that $\iint_{\Delta(L) \rho^{2}} d A=\iint_{\Delta^{\prime}(L)} d A$ we obtain

$$
\begin{aligned}
& -2 \pi \Re\left\{\sum_{j=1}^{s} \alpha^{(j)} \log a^{(j)}\right\}+2 \pi \Re\left\{\sum_{j=s+1}^{r}\left|\alpha^{(j)}\right| \log a^{(j)}\right\}+o(1) \\
& \geqq 4 \pi \Re\left\{\sum_{j=s+1}^{r}\left|\alpha^{(j)}\right| e^{i \phi_{i}} \cos \phi_{j} \log a^{(j)}\right\}+2 \pi \Re\left\{\sum_{j=r+1}^{n} \alpha^{(j)} a_{m_{j}-3}^{(j)}\right\}+o(1) .
\end{aligned}
$$

The explicit terms occurring are independent of $L$ and an elementary calculation gives

$$
\Re\left\{\sum_{j=1}^{r} \alpha^{(j)} \log a^{(j)}+\sum_{j=r+1}^{n} \alpha^{(j)} a_{m_{j-3}}^{(j)}\right\} \leqq 0
$$

which is just the fundamental inequality (2).

As regards the possibility of equality in (2) we first observe that by a standard type of argument [10] every trajectory or portion thereof lying in the intersection of an end, strip, or circle domain with $\Delta$ must be mapped by $f$ into another such, the mapping being isometric in the $Q$-metric. In view of the condition for equality in Teichmüller's result III the same must be true in the set $\mathfrak{M}$ and in the intersection of any ring domain with $\Delta$. Further no open set on $\Re$ can be left uncovered by the image $\Delta^{\prime}$ of $\Delta$ under $f$ and the boundary of $\Delta^{\prime}$ relative to $\Re$ must consist of a finite number of arcs on trajectories or on trajectories closed by a point of $C$. We have so far considered only the modified surface $\Re$ on which there are no simple poles but it is clear that the above conditions on it imply the analogous statements for the original surface.

If a pole $P_{j}, j>r$, of order $>2$ exists the prescribed form of the mapping $f$ there combined with the above conditions implies immediately that $f$ is the identical mapping in a neighborhood of $P_{j}$ and thus everywhere in $\Delta$. A similar remark applies if there is a pole $P_{j}, j \leqq r$, of order 2 for which the corresponding coefficient $a^{(j)}=1$.

If a pole of order 1 exists interior to $\Delta$ it must be mapped into itself and the remaining conditions imply at once that $f$ is the identical mapping in a neighborhood of this point and so everywhere. 
Suppose a pole $P$ of order 1 exists on a slit boundary of $\Delta$ but part of the trajectory bearing the slit is interior to $\Delta$. Then the homotopy condition on $f$ implies that $P$ lies in the component of the complement of $\Delta^{\prime}$ corresponding to the slit. It is readily seen that this must coincide with the original slit in view of the isometric property of $f . \Delta$ can then be enlarged to include this slit and $f$ extended to have the same properties as before. That $f$ is the identity then follows from the preceding paragraph.

Finally if we have a double pole $P_{j}$ with $\alpha^{(j)}<0, P_{j}$ will lie in a circle domain. If the circle domain is proper, i.e. has a singular point on its boundary, we see at once that it must be mapped onto itself so that $\left|a^{(j)}\right|=1$. The alternative case can occur only in the following situations:

(a) $\Re$ the sphere, $Q(z) d z^{2}$ a hyperelliptic differential whose only singular points are two poles of order 2 .

(b) $\Re$ the sphere, $Q(z) d z^{2}$ a hyperelliptic differential whose only singular points are one pole of order 2 and two poles of order 1 , the two latter being limiting end points of a single trajectory, $\Delta$ obtained from $\Re$ by slitting at least along this trajectory.

(c) $\Re$ a simply-connected hyperbolic surface, $Q(z) d z^{2}$ a positive quadratic differential on $\Re$ whose only singular point is a pole of order 2 .

In saying $\Re$ is the sphere, we mean $\Re$ is a closed surface of genus zero, i.e. conformally equivalent to the sphere. The same interpretation is to be attached to the term in the statement of the Main Theorem, and an analogous one to the term "linear transformation."

These results follows at once from the familiar formula for the algebraic sum of the orders of poles and zeros of a positive quadratic differential together with a simple topological observation in the case of (b). It is verified directly that for equality in (2) we must have $|a|=1$ for the coefficient $a$ at the pole in cases (b) and (c). In case (a) we can have equality if and only if $f$ is a linear transformation with the two poles as fixed points.

This completes the proof of the Main Theorem.

9. The Main Theorem can readily be extended in the following manner:

EXTENDED THEOREM. Let the conditions be the same as in the Main Theorem except that now the quadratic differential is only required to be regular on the boundary of $\Re$. Further $f$ is required to admit a continuous extension to any maximal open boundary arc or complete boundary of $\Re$ on which $Q(z) d z^{2} \leqq 0$ and to map such an arc or boundary into itself, the homotopy $F(P, t)$ is to admit a similar extension and also to satisfy a similar mapping condition for each given $t$. Then the same conclusions obtain as in the Main Theorem.

It follows at once that $f$ is actually a homeomorphism into on the above arcs and boundaries and $f$ is analytic thereon in terms of boundary uniformizers. The Extended Theorem is most easily proved by "doubling" the surface $\Re$ across those boundary arcs and boundaries on which $Q(z) d z^{2} \leqq 0$. 
The quadratic differential extends by reflection to give a positive quadratic differential on the new surface and the entities corresponding to $\Delta, f$, and $F$ are obtained in a standard manner. The Main Theorem in its original form applies to the new surface and we obtain at once the Extended Theorem.

It should be observed that the Extended Theorem can be proved directly in the same manner as the Main Theorem. Indeed we can obtain a decomposition of $\Re$ relative to the more general type of quadratic differential analogous to that given in the Basic Decomposition Theorem for a positive quadratic differential and the remainder of the proof carries over with only trivial modifications.

10. At the end of $\S 1$ we remarked that the theorem to be proved includes the great majority of the results of geometric function theory. Among these let us mention particularly the extremal properties of certain normal maps of multiply-connected domains [21], the Koebe 1/4 Theorem, the bounds for the modulus of a normalized regular univalent function in the unit circle, the distortion theorem, generalizations of these to bounded regular univalent functions [19] and to circular rings [1], the fundamental inequalities in the parallel slit theorem, Grötzsch's distortion [2] and displacement [4] theorems, and Teichmüller's coefficient result [25].

Indeed, in each of these situations the extremal domain is an admissible domain for a (positive) quadratic differential either on the sphere or on a circular domain and the competing functions provide just admissible mappings. In all the above cases the homotopy condition is trivially satisfied. In results such as Grötzsch's distortion and displacement theorems there is in general in addition to the fundamental inequality an auxiliary result which provides the exact domain of variation of some entity. This auxiliary result usually depends on some special explicit consideration and does not come under the general theorem.

Moreover there are many results which while not directly included in the enunciation of the theorem are easy consequences of the method employed in its proof. Among these we may mention the extremal properties of certain other normal maps of multiply-connected domains $[1 ; 21]$, Komatu's generalization of Löwner's Lemma [18], the results concerning the closest boundary point on equally spaced half-rays for the image by normalized univalent mappings of the unit circle and their generalization to circular rings $[8 ; 20]$. The present approach makes particularly clear why in the case of more than one ray we can allow the functions considered to be meromorphic while in the case of the Koebe $1 / 4$ Theorem and its generalizations the functions must be regular, a fact first pointed out by Rengel [20].

Moreover combining the above methods with certain other ideas [11] we can obtain proofs of Schwarz's Lemma, Löwner's Lemma, the BieberbachEilenberg Theorem, and various other results.

Finally it should be remarked that the Main Theorem can be applied in 
the coefficient problem for multiply-connected domains [24] in the same manner that Teichmüller's coefficient result has been applied for simplyconnected domains [23].

11. We conclude with two remarks. Remark 1. The above Main and Extended Theorems and the other applications of the method employed indicated above by no means exhaust the scope of Teichmüller's principle enunciated in $\$ 1$. For other illustrations see $[12 ; 13 ; 14 ; 15]$.

REMARK 2. Royden [22] has recently written a paper which has some relation to the present one. In the case where poles of order $>1$ are present his result becomes a weak form of the equality statement (i) or (ii) of the Main Theorem, i.e. instead of assuming merely that the left-hand side of (2) vanishes he assumes that every term in the expression of which we take the real part vanishes. In the case where no pole of order $>1$ is present his result can be derived in a straightforward manner from the results of Teichmüller [26]. In his formulation Royden allows a homotopy of a priori more general type than that stated here. However it can be readily modified to give one of the latter type so there seems to be for the present purpose no advantage in the apparently more general formulation.

\section{BIBLIOGRAPHY}

1. H. Grötzsch, Über einige Extremalprobleme der konformen Abbildung, Berichte über die Verhandlungen der Sächsischen Akademie der Wissenschaften zu Leipzig, MathematischPhysische Klasse, I and II, vol. 80 (1928) pp. 367-376 and 497-502.

2. - Über die Verzerrung bei schlichter konformer Abbildung mehrfach zusammenhängender schlichter Bereiche, ibid. I and II, vol. 81 (1929) pp. 38-47 and 217-221, III, vol. 83 (1931) pp. 283-297.

3. - Zum Parallelschlitztheorem der konformen Abbildung schlichter unendlich-vielfach zusammenhängender Bereiche, ibid. vol. 83 (1931) pp. 185-200.

4. - Über die Verschiebung bei schlichter konformer Abbildung schlichter Bereiche, ibid. I, vol. 83 (1931) pp. 254-279 and II, vol. 84 (1932) pp. 269-278.

5. —— Über zwei Verschiebungsprobleme der konformen Abbildung, Preuss. Akad. Wiss. Sitzungsber. (1933) pp. 87-100.

6. - Die Werte des Doppelverhältnisses bei schlichter konformer Abbildung, ibid. pp. 501-514.

7. - Über die Geometrie der schlichten konformen Abbildung, ibid., I and II (1933) pp. 654-671, 893-908, III (1934) pp. 434-444.

8. - Einige Bemerkungen zur schlichten konformen Abbildung, Jber. Deutschen Math. Verein. vol. 44 (1934) pp. 270-275.

9. James A. Jenkins, Some problems in conformal mapping, Trans. Amer. Math. Soc. vol. 67 (1949) pp. 327-350.

10. - Remarks on "Some problems in conformal mapping," Proc. Amer. Math. Soc., vol. 3 (1952) pp. 147-151.

11. - Some results related to extremal length, Contributions to the Theory of Riemann Surfaces, Annals of Mathematics Studies, no. 30, 1953, pp. 87-94.

12. - Symmetrization results for some conformal invariants, Amer. J. Math. vol. 75 (1953) pp. 510-522.

13. - On a recent note of Kolbina, Duke Math. J. vol. 21 (1954) pp. 155-162. 
14. —— On Bieberbach-Eilenberg functions, Trans. Amer. Math. Soc. vol. 76 (1954) pp. $389-396$.

15. - On a problem of Gronwall, Ann. of Math. vol. 59 (1954) pp. 490-504.

16. James A. Jenkins and Marston Morse, Curve families $F^{*}$ locally the level curves of a pseudoharmonic function, to appear in Acta Math.

17. James A. Jenkins and D. C. Spencer, Hyperelliptic trajectories, Ann. of Math. vol. 53 (1951) pp. 4-35.

18. Y. Komatu, Untersuchungen über konforme Abbildung von zweifach zusammenhängenden Gebieten, Proceedings of the Physico-Mathematical Society of Japan vol. 25 (1943) pp. $1-42$.

19. G. Pick, Über die konforme Abbildung eines Kreises auf ein schlichtes und zugleich beschränktes Gebiet, Kaiserliche Akademie der Wissenschaften in Wien, Mathematisch-naturwissenschaftliche Klasse, Sitzungsberichte Abt II a, vol. 126 (1917) pp. 247-263.

20. E. Rengel, Über einige Schlitztheoreme der konformen Abbildung, Schriften des Mathematischen Seminars und des Instituts für angewandte Mathematik der Universtität Berlin vol. 1 (1933) pp. 141-162.

21. - Existenzbeweise für schlichte Abbildungen mehrfach zusammenhängender Bereiche auf gewisse Normalbereiche, Jber. Deutschen Math. Verein. vol. 44 (1934) pp. 51-55.

22. H. Royden, The conformal rigidity of certain subdomains on a Riemann surface, Trans. Amer. Math. Soc. vol. 76 (1954) pp. 14-25.

23. A. C. Schaeffer and D. C. Spencer, Coefficient regions for schlicht functions, Amer. Math. Soc. Colloquium Publications, vol. 35, 1950.

24. M. Schiffer and D. C. Spencer, The coefficient problem for multiply connected domains, Ann. of Math. vol. 52 (1950) pp. 362-402.

25. O. Teichmüller, Ungleichungen zwischen den Koeffizienten schlichter Funktionen, Preuss. Akad. Wiss. Physikalisch-Mathematische Klasse, 1938, pp. 363-375.

26. - Extremale quasikonforme Abbildungen und quadratische Differentiale, Abhandlungen der Preussischen Akademie der Wissenschaften, Mathematisch-naturwissenschaftliche Klasse, 1939, No. 22.

27. — Über Extremalprobleme der konformen Geometrie, Deutsche Mathematik vol. 6 (1941) pp. 50-77.

The Johns Hopkins University, BALTimore, MD. 